\title{
Injerencia del modelo burocrático-populista en la reforma universitaria. Caso Universidad del Zulia
}

\author{
Pereira Burgos, Morela* \\ Díaz Barrios, Jazmín** \\ Díaz Nava, María Gabriela***
}

\section{Resumen}

La educación superior enfrenta el desafío de adecuarse a las condiciones de una realidad distinta, lo que implica realizar importantes ajustes orientados a alcanzar una mayor pertinencia interna y externa de sus resultados. En tal sentido, cobra relevancia el estudio de los modelos de gestión que han dominado los procesos de dirección y por ende determinado los objetivos, estrategias y políticas de desarrollo de la triada docencia-investigación-extensión del ente universitario. Partiendo de estas premisas, el presente trabajo tiene como objetivo analizar los modelos de gestión en la Universidad del Zulia (LUZ) y su relación con la reforma universitaria durante el período 1988-2004. Desde el punto de vista metodológico, la investigación es de tipo documental utilizando la hermenéutica como herramienta de análisis. Los resultados muestran que el modelo de gestión imperante ha sido el burocrático-populista; aspectos como toma de decisiones con escasa o nula participación de los diversos actores, planificación centralizada y carente de coordinación, exceso de formalización, lentitud en las respuestas, entre otros, han dado lugar a una institución cuyos cambios son casi imperceptibles, en algunos casos extemporáneos y en el peor de los casos no se muestra proclive al mismo.

Palabras clave: Modelo de gestión, reforma universitaria, Universidad del Zulia, modelo burocrático-populista.

Recibido: 06-10-11. Aceptado: 18-07-12

* Doctora en Ciencias Sociales, mención gerencia, MgSc en Gerencia de Empresas, Mención Servicios Administrativos. Profesora e Investigadora del CEE en FCES/LUZ. Acreditada en el Programa de Estímulo a la Innovación e Investigación (PEII), e-mail: morela.pereira@gmail.com

** Doctora en Ciencias Gerenciales. Post Doctorado en Investigación y Gerencia. Investigadora del CEE y docente en la FCES-LUZ. Acreditada en el Programa de Estímulo a la Innovación e Investigación (PEII), e-mail: jazdibar@gmail.com

*** MgSc en Gerencia Pública. Profesora en la Universidad Rafael Urdaneta. Auxiliar de Investigación del (CEE). Facultad de Ciencias Económicas y Sociales (FCES). Universidad del Zulia (LUZ). Acreditada en el Programa de Estímulo a la Innovación e Investigación (PEII), e-mail: gabydn80@yahoo.es 


\title{
Interference of the Bureaucratic-Populist Model in University Reform. Case of the University of Zulia
}

\begin{abstract}
Higher education faces the challenge of adapting itself to the conditions of a different reality that involves making significant adjustments focused on achieving greater internal and external relevance in its results. In this sense, the study of management models that have dominated directorial processes and, therefore, the objectives, strategies and policies for developing the teaching-research-extension triad of the university entity, becomes relevant. The objective of this work is to analyze management models at the University of Zulia (LUZ) and their relation to university reform during the 1988-2004 period. From the methodological viewpoint, research is of the documentary type using hermeneutics as a tool for analysis. Results show that the dominating management model has been the bureaucraticpopulist model. Aspects such as making decisions with scant or no participation by the diverse actors, centralized planning that lacks coordination, an excess of formalization, slow responses, among others, have brought about an institution whose changes are almost imperceptible, in some cases, extemporaneous, and in the worst cases, do not demonstrate a proclivity to change.
\end{abstract}

Keywords: Management model, university reform, University of Zulia, bureaucratic-populist model.

\section{Introducción}

La crisis universitaria es un tema largamente debatido en el ámbito mundial, la sociedad como un todo demanda de estas casas de estudio formas innovadoras de abordar sus funciones fundamentales -docencia, investigación, extensión- en términos de calidad, pertinencia y equidad. En palabras de Lanz y Fergusson (2005) el actual modelo educativo es inviable ya que no se corresponde con las expectativas de los nuevos actores, con las nuevas condiciones de la sociedad de la información, con las nuevas exigencias de un mundo globalizado.

Se espera de las instituciones universitarias la formación de profesionales, desarrollo de nuevos conocimientos, y soluciones a los problemas ingentes de la sociedad, así como su participación acti- va en la definición de los lineamientos y estrategias que guíen el desarrollo del país. En suma, su reto fundamental es el de reformarse.

En Venezuela, tanto el Estado como las universidades autónomas han venido ofreciendo alternativas dirigidas a la renovación de la institución. Destaca el papel de estas últimas en los procesos de cambio, pues ante la indudable redefinición del panorama mundial y local han asumido como necesidad urgente la reforma en las maneras de pensar, sentir y hacer de la institución universitaria.

En este contexto, cobra relevancia el estudio de los modelos de gestión que han dominado los procesos de dirección y por ende determinado los objetivos, estrategias y políticas de desarrollo de la triada docencia-investigación-extensión de la institución universitaria. En el caso 
Injerencia del modelo burocrático-populista en la reforma universitaria..

Pereira Burgos, Morela; Díaz Barrios, Jazmín y Díaz Nava, María Gabriela

de la Universidad del Zulia (LUZ), resulta de interés el estudio de la gestión del proceso de reforma que desde hace algunos años viene adelantando la institución, como resultado de las presiones y críticas recibidas desde los ámbitos interno y externo.

Partiendo de lo anterior el presente trabajo se plantea como objetivo analizar los modelos de gestión en LUZ y su relación con la reforma universitaria durante el período 1988-2004. Desde el punto de vista metodológico, la investigación es de tipo documental utilizando la hermenéutica como herramienta de análisis.

\section{Administración, gerencia y gestión}

En la generalidad de los casos los conceptos de administración, gerencia y gestión se emplean como sinónimos para hacer referencia al conjunto de prácticas organizacionales dirigidas a alcanzar sus objetivos. De acuerdo con Ochoa et al. (2007), es común observar en la práctica que el término management es traducido como administración pero también como gerencia.

Para Chiavenato (2001:3) la palabra administración proviene del latín (ad, dirección hacia, tendencia: minister, comparativo de inferioridad, y el sufijo ter, que indica subordinación u obediencia, es decir que cumple una función bajo el mando de otro, quien le presta un servicio a otro) y significa subordinación y servicio. Agrega el autor que la administración es el proceso de planear, organizar, dirigir y controlar el empleo de los recursos organizacionales para conseguir determinados objetivos con eficacia y eficiencia.
Gerencia refiere, según la Real Academia Española (2001), al cargo que ocupa el gerente, es decir la persona que lleva la gestión administrativa de una empresa o institución. Por tanto, es un proceso que implica la coordinación de todos los recursos disponibles en una organización (humanos, físicos, tecnológicos, financieros), para que a través de los procesos de planificación, organización, dirección y control se logren objetivos planteados.

Por su parte, el concepto de gestión de acuerdo con la Real Academia Española (2001) proviene del latín gesio y hace referencia a la acción y al efecto de gestionar o de administrar. Se trata de un proceso emprendido por una o más personas para coordinar las actividades laborales de otros individuos. También puede entenderse como la capacidad de la institución para definir, alcanzar y evaluar sus propósitos, con el adecuado uso de los recursos disponibles. La noción implica además acciones para gobernar, dirigir, ordenar, disponer u organizar. De esta forma, la gestión supone un conjunto de trámites que se llevan a cabo para resolver un asunto, concretar un proyecto o administrar una empresa u organización.

Algunos autores contemporáneos señalan como diferencia fundamental entre estos conceptos el alcance de cada uno. A la gerencia, le otorgan una connotación más externa, innovadora y de mayor valor agregado en contraste con la administración que se le considera más interna, más del manejo de lo existente o básicamente funcional. Otros estudiosos platean a la administración como un proceso llevado a cabo en las organizaciones para cumplir sus objetivos, es decir 
como fenómeno general, mientras que la gerencia se concibe como un tipo particular de administración, cuyo objetivo final es la eficiencia.

Por su parte, la gestión es planteada según Mora (1999), como una función institucional global e integradora de todas las fuerzas que conforman una organización. En ese sentido, cobra singular importancia el ejercicio del liderazgo como factor clave de la dirección. En suma, lo fundamental está en considerar que la administración, gerencia o la gestión asumen el proceso administrativo como un todo indisoluble. De esta manera, para efectos del presente trabajo se empleará el término gestión como sinónimo de administración y/o gerencia.

\section{Modelos de gestión}

Entender la misión, objetivos y direccionamiento de las organizaciones implica conocer el modelo de gestión que guía la toma de decisiones y determina, por tanto, el quehacer diario y futuro de las mismas. En este sentido es fundamental definir el constructo modelo de gestión.

El término modelo utilizado en distintos ámbitos y con diversos significados proviene del concepto italiano modello (Real Academia Española, 2001). Ahora bien aplicado al campo de las ciencias sociales, un modelo hace referencia al arquetipo que, por sus características idóneas, es susceptible de imitación o reproducción. Lo anterior unido al concepto de gestión previamente definido, hace referencia a un esquema o marco de referencia para la administración de una organi- zación ya sea en el ámbito público o privado.

Los modelos de gestión responden a las posturas teóricas, cuyos fundamentos han pretendido explicar el fenómeno organizacional aportando soluciones de acuerdo al contexto histórico referencial en el cual cada uno de ellos surgió. Desde el punto de vista de la teoría administrativa, Chiavenato (2001) plantea que los modelos se han ido desarrollando teniendo como eje distintos aspectos de la administración: tareas, estructura, personas, tecnología y ambiente, han sido en su momento el pivote alrededor del cual ha girado la gestión organizacional.

En el ámbito público, estos modelos han coexistido de manera paralela desarrollándose con un estilo particular: la estructura ha tomado la forma de la burocracia, el énfasis en las personas se ha expresado a través de la tecnocracia, y en el espacio latinoamericano, todo lo anterior ha sido matizado con las tendencias populistas que han impregnado el aparato estatal y por ende su gestión.

En palabras de Weber (1964) la burocracia designa un cuerpo profesional de funcionarios, organizado en forma jerárquica piramidal, que sigue reglas $y$ procedimientos uniformes e impersonales. En este orden de ideas, Subirats (1989) plantea que la burocracia implica al conjunto de personas organizadas de manera jerárquica que realizan un trabajo rutinario, repetitivo cuyas pautas de trabajo están fuertemente normadas y fijadas mediante procedimientos. En ella se produce una clara distinción entre la cúpula decisional y el núcleo de operaciones, con fuertes dosis de control que evite 
Injerencia del modelo burocrático-populista en la reforma universitaria..

Pereira Burgos, Morela; Díaz Barrios, Jazmín y Díaz Nava, María Gabriela

las desviaciones o incumplimientos, limitando la discrecionalidad.

Precisa además el autor, que el control, y por ende el poder, estará en función de la distribución de los recursos, como información, dinero, tiempo, entre otros. Por otra parte, el control exhibe diversas formas considerando los flujos de información, manejo de recursos/servicios y las presiones de grupos de clientelas o interés por conseguir más recursos a cambio de concesiones y respaldos.

Ahora bien, en el aparato público la praxis burocrática, a lo largo de su existencia, se ha ido deformando de manera tal que los ideales weberianos básicos han desaparecido o están mediatizados y progresivamente substituidos, por la burocratización. Término que puede ser asociado a lo que Malavé y Piñango (2004) denominan, connotaciones negativas de la burocracia haciendo referencia a sus males, excesos y peso insoportable, así como a la rigidez, ineficacia y deshumanizado trato.

Otro modelo de gestión que irrumpe en la escena latinoamericana, y especialmente del país al consolidarse el proyecto democrático, fue el denominado tecnocrático. En palabras de Ochoa (1995), la organización tecnocrática es aquella cuya dirección está en poder de los técnicos y donde el criterio básico de dirección es la racionalidad económica con base en la eficiencia de las instituciones. Plantea Guerrero (2006:18) que desde la década del sesenta las sociedades políticas occidentales han mostrado una tendencia: al mismo tiempo que son gobernadas democráticamente, han comenzado a brindar espacio a las fuerzas detentadoras de los medios de operación política, como los grupos de presión, así como los técnicos, con su creciente influencia en los asuntos públicos.

Siendo que la tecnocracia funciona dentro de la burocracia, de acuerdo con Kelly (2004), es un fenómeno asociado a la fusión entre el poder y el conocimiento técnico. Agrega la autora, que en las burocracias formales el cuerpo de expertos en los asuntos del Estado debe garantizar el buen funcionamiento de los servicios públicos y prestar asesoría especializada en la formulación de políticas.

El poder es detentado por los más capaces, aquellos que han accedido a él considerando su preparación y capacidades. La meritocracia emerge como la vía para hacer carrera dentro de la administración pública, a la par de enfrentar los ascensos de la burocracia disfuncional, sustentados en el clientelismo y la antigüedad. Este modelo, pareciera estar orientado a superar la rigidez del paradigma weberiano, mediante la flexibilización organizativa e introduce conceptos como calidad y productividad en la búsqueda de eficacia y eficiencia en las organizaciones.

A partir del proceso democrático, en Venezuela, surge, articulado al modelo burocrático, el populismo. Kelly (2004:22), lo define como la tentación de los gobiernos a implantar políticas de corte popular para complacer a los votantes. Agrega Burbano (1998), que el populismo está asociado a fenómenos como: tipo de retórica, figura de líder y su carisma, vinculo emocional y cuasi-religioso entre las masas y el líder, cultura política y clientelismo, este último como mecanismo de intercambio entre el líder y sus seguidores. 
En palabras de Pereira et al. (2008), el populismo es el ejercicio de la política a través de una oferta atractiva a las masas, con el fin de manipularlas y a la vez legitimarse y permanecer en el poder. Por su parte, Ochoa et al. (1996:41) conjugan estos dos fenómenos en lo que denominan modelo "burocrático-populista" para denotar la conducción del aparato público, basada en la colaboración de clases, a través del gasto público, para el logro de los objetivos. Agregan Pereira et al. (2008) que la administración burocrática-populista, se caracteriza por contar con grandes estructuras organizativas con altos niveles jerárquicos y numerosos departamentos propios del modelo Weberiano; a la par del ejercicio de una gestión clientelar.

\section{Modelos de Gestión Universitaria: El caso de LUZ}

En las universidades de la región latinoamericana, los modelos de gestión se han expresado con ciertas particularidades a lo largo de su evolución. Así tenemos, de acuerdo con Morles et al. (2003), que la universidad tradicional latinoamericana de fuerte inclinación religiosa, basada en la especulación teórica, la retórica y el intelectualismo, fue luego sustituida por la napoleónica, de escuelas aisladas y formación profesional especializada, cuyo modelo de gestión se basó primero en las tareas y luego en la estructura, con influencias de otras prácticas gerenciales variadas.

Específicamente, dentro de los modelos estructurales, la napoleónica tenía un modelo burocrático, vale decir, donde prima la extrema división del trabajo, con grandes estructuras organizativas, numerosos niveles jerárquicos y departamentos, clara asignación de tareas especializadas a cada trabajador y gran centralización de las decisiones. Por su parte, el modelo tecnocrático, según Esté (1998), también tuvo su aporte en nuestras universidades. En cuanto al modelo burocrático-populista, plantea Pereira et al. (2008) que nuestras universidades públicas, exhiben todas las características de este modelo: una suerte de esquema rígido y centralizado, sin las bondades teóricas de la burocracia, más bien, matizado con un clientelismo y discrecionalidad sin reservas.

Se encuentra una coexistencia de los modelos burocrático, tecnocrático y populista en la gestión académica-administrativa. Cada uno aportando características propias que configuran un modelo de gestión que si bien no es exclusivo, se ve fuertemente influenciado por las particularidades que le confiere la autonomía universitaria.

El modelo de gestión burocrático ha estado presente a lo largo de la historia de las universidades autónomas. Sin embargo, los cambios contextuales han ido permeando su estructura configurando una institución que lejos de acercarse al modelo ideal weberiano, paulatinamente ha desarrollado las disfuncionalidades de la burocratización. Tal es el caso del crecimiento vertiginoso que experimentó la educación superior en la década del 60 con el advenimiento del proceso democrático. En palabras de Pereira et al. (2008), la masificación contribuyó a la burocratización de la institución universitaria, manifestada entre otros aspectos, en la presencia exagerada de nor- 
Injerencia del modelo burocrático-populista en la reforma universitaria..

Pereira Burgos, Morela; Díaz Barrios, Jazmín y Díaz Nava, María Gabriela

mas y reglas que rigen los procesos académicos y administrativos, los cuales en muchos casos se constituyen en obstáculos que interfieren en el normal desarrollo de las actividades.

Entre otros aspectos, se observa, abultamiento de las nóminas de docentes, empleados y obreros que conlleva a destinar la mayor parte del presupuesto asignado al pago de personal; procesos lentos y complejos resultado del número de instancias por las que se debe tramitar cualquier requerimiento. Una lucha constante de los diversos actores involucrados en el quehacer académico por alcanzar y mantener el poder, y por tanto el control de los centros de decisión estratégicos.

En cuanto al modelo tecnocrático en la educación pública superior, éste logró impactar algunos procesos clave para la articulación universidad-sector productivo, en el marco del modelo de sustitución de importaciones, al promover reformas orientadas a la formación de profesionales que respondieran a las necesidades de desarrollo tecnológico. Sin embargo, no logra imponerse, en las universidades nacionales, debido a la oposición de sectores profesorales defensores del modelo humanístico liberal, quienes argumentaban de acuerdo con López (1996) que la institución estaba perdiendo por una parte, su papel crítico-transformador, y por otra su vinculación con la problemática social al ponerse al servicio del mercado.

Esta oposición se constituyó en un factor importante, que orientó al Estado a crear y promover nuevas instituciones de educación superior donde el modelo tecnocrático tuviera cabida. Tal es el caso de las universidades experimentales y la proliferación de iniciativas del sector privado. En este sentido, señala Esté (1998), que los cambios en torno a la estructura productiva, llevan a una nueva estrategia educativa, en la que en forma más coherente, se observan rasgos del modelo tecnocrático, inscritos en las reformas propuestas por los organismos internacionales de cooperación y asistencia educativa a nivel mundial.

Por su parte, el modelo burocrático-populista, en la Universidad Autónoma venezolana, centro de formación e investigación por excelencia en el país, se caracteriza por su independencia para elegir su gobierno y cogobierno, así como por su complejidad estructural y procesal. Pereira et al. (2008: 619-620) manifiestan que se expresa o materializa a través de una toma de decisiones centralizada, una planificación legitimadora, utilización de los gremios sólo con fines socio-económicos, politización que sustenta el clientelismo, excesiva formalización, alta verbalización no traducida en acciones y falta de profundidad en el análisis.

Este conjunto de elementos, que forman parte de la gestión institucional, fueron estudiados por Pereira et al. (2008), dando como resultado las siguientes dimensiones para el modelo burocrático-populista: toma de decisiones, planificación, mecanismos de asociación, mecanismos de regulación, crecimiento, evaluación, selección de personal, criterios de dirección, elementos del discurso y manejo de conflictos. A continuación se realiza una breve caracterización de aquellos considerados relevantes para el tema estudiado: 
- Toma de decisiones: relacionada directamente con el nivel de centralización o descentralización, implica el grado en que las decisiones se concentran en una parte de la organización.

- Planificación: procedimiento que implica la selección de misiones y objetivos y de las acciones para cumplirlos, y requiere de la toma de decisiones para optar entre diferentes cursos de acción futuro.

- Mecanismos de asociación: Los grupos sociales considerando sus expectativas, necesidades o intereses tienden a integrarse mediante diversos mecanismos entre los cuales destacan gremios, sindicatos, partidos políticos, etc.

- Mecanismos de regulación: concerniente al proceso de control, el establecimiento de normas, medición del desempeño, que buscan influenciar la conducta de la gente que trabaja en la organización.

- Crecimiento: La hipertrofia burocrática que aqueja a la administración pública venezolana. Adicionalmente, debido a la rigidez burocrática, en vez de enriquecer los cargos, añadiéndole otras funciones, se tiende a crear unidades para cada necesidad sin análisis previo de viabilidad e incluso a la medida de las necesidades individuales.

- Criterio de dirección: La dirección supone el logro efectivo de todo lo planeado mediante la autoridad ejercida a base de decisiones.

- Elementos del discurso y manejo de conflictos: Profusión de documentos, discurso retórico, mediático, sobre los problemas medulares sin concreción. No hay discusiones, los análisis permanecen en la superficie que no compromete.

En concreto, de acuerdo a los planteamientos anteriores, puede decirse que el modelo de gestión predominante ha sido el burocrático con matices del modelo Tecnocrático y elementos del fenómeno populista. Sin embargo, se tiene que todos intervienen en la gestión universitaria venezolana, específicamente en las universidades autónomas.

En el caso de la Universidad del Zulia, como universidad autónoma, la situación no es distinta y por tanto la gestión de sus procesos ha estado sujeta a la combinación de estos modelos. Entre esos procesos influidos por el modelo burocrático-populista imperante, resulta de interés el estudio de la gestión del proceso de reforma que desde hace algunos años viene adelantando la institución como resultado de las presiones y críticas recibidas tanto a lo interno como a lo externo de la institución.

\section{Reforma universitaria en LUZ, período 1988-2004}

El término reforma es un vocablo que presenta un carácter polisémico por cuanto atendiendo a su objetivo o ámbito de acción connota diversos significados. Al respecto plantea Lanz (2004:200) que no existe un atributo preciso para esta palabra, ni mucho menos una elección consciente en relación al universo semiológico donde habita todo un repertorio conceptual usado de modo intercambiable: puede sustituirse sin problemas por cambio, transformación o renovación. 
Injerencia del modelo burocrático-populista en la reforma universitaria..

Pereira Burgos, Morela; Díaz Barrios, Jazmín y Díaz Nava, María Gabriela

Ahora bien, una reforma puede abordarse de distintas maneras. Burke y Litwin, citado por French \& Bell (1996) plantean los cambios revolucionarios (cambian los cimientos de la organización) y los evolutivos (cambio de características, no de la esencia de la organización). Hussey (1997) en la misma tónica, indica dos tipos de cambios: incrementales (se avanza mediante la evolución y no la revolución) y fundamentales (cambios amplios, afectan drásticamente las operaciones e implican trastornos importantes).

En el contexto de la presente investigación la reforma universitaria se expresa como un proceso incremental de modificaciones, en todos los órdenes de la institución, que implican las funciones básicas de la universidad. Para lograr lo anterior, se revisarán las decisiones del Consejo Universitario de la Universidad del Zulia, reseñadas en las gacetas oficiales durante el periodo estudiado.

Considerando lo anterior, cabe destacar que la Universidad del Zulia, de acuerdo con las gacetas del Consejo Universitario publicadas a lo largo del período estudiado (1988-2004) presentó sesenta y cinco (65) propuestas de reforma en distintos órdenes: trece (13) académicas, una (1) administrativa, nueve (9) estructurales, y cuarenta y dos (42) legales. Además estas fueron de distintos alcances: unas con cambios puntuales y/o superficiales; otras plantearon modificaciones profundas y/o de gran amplitud, en consonancia con el clima reformador que ocupó el ámbito latinoamericano desde la década de los ochenta así como en respuesta a las presiones recibidas desde el entorno e incluso desde su seno.
Sin embargo, las propuestas e iniciativas reformadoras no siempre terminan de concretarse, quedando algunas sólo en buenos proyectos. Esta situación pareciera estar asociada, en muchos casos, a la injerencia del modelo de gestión de la institución universitaria. De acuerdo con Lanz (2004:208) intenciones, propósitos e intereses sobran, lo que ha faltado históricamente es la capacidad para traducir esas políticas en vida universitaria real.

\section{Elementos del modelo burocrático-populista en la reforma de LUZ}

Se parte del supuesto de que el modelo de gestión dominante en la Universidad del Zulia, considerando para ello el planteamiento de Pereira et al. (2008), durante el período de estudio, es el burocrático-populista con rasgos del modelo tecnocrático. El análisis abarcará las cuatro gestiones rectorales, es decir será global, respecto a los elementos que caracterizan el modelo en las universidades autónomas del país.

Desde el punto de vista de la toma de decisiones, se observa que las propuestas de reforma son formuladas desde las máximas instancias de decisión con escasa o nula participación de la comunidad universitaria, es decir, profesores, alumnos, personal administrativo y obrero. Muchas de ellas tienen un carácter unilateral porque se diseñan, planifican, elaboran e incluso se ponen en práctica considerando sólo a grupos minoritarios que en determinados momentos detentan el poder, generando resistencia y por ende rechazo al cambio emprendido. 
En palabras de Fergusson (2003:48) con frecuencia las autoridades producen grandes resoluciones de organismos de dirección en las que las reformas aparecen "decretadas", dando como resultado que estos mandatos sean sistemáticamente desoídos no sólo por la poca legitimidad del gobierno universitario sino también por la impronta demagógica y oportunista que rodea a este tipo de proclama.

La situación planteada se refuerza, por la apatía y desinterés del universitario, quien ante el llamado a participar en jornadas, talleres y/o conferencias relativas al tema asume una postura distante $y$, aún cuando en otros casos pudiera mostrarse interesado, en la práctica sus acciones no lo reflejan. Al respecto señala Parra (1996:491) que el docente universitario está consciente de la necesidad de que se produzcan cambios, pero a la vez, mantiene inalterados los esquemas que sustentaron el actual modelo de universidad.

En la toma de decisiones, además intervienen aspectos políticos que en algunos casos determinan la aprobación o no de la reforma. Tal es el caso del planteamiento de reforma presentado en la gestión 2000-2004 relativa a la separación de poderes del gobierno universitario, la cual a pesar de ser una propuesta novedosa en cuanto a gestión, no se concretó, posiblemente por falta de voluntad política y el costo que pudiera representar. Tal como plantea Lindblom (1991:7) en toda política pública se asiste a un conjunto de procesos, decisiones, resultados sin excluir los conflictos entre intereses presentes en cada momento, tensiones entre diferentes racionalidades or- ganizativas y de acción, y entre diferentes baremos y perspectivas evaluadoras. Se trata de un panorama lleno de poderes en conflicto, enfrentándose y colaborando ante opciones y cursos de acción específicos.

Por su parte, la Planificación de tales propuestas en consonancia con el resto de los procesos de planificación de la institución universitaria exhibe la desarticulación entre los planificadores y los ejecutores. En la generalidad de los casos el plan funcional para los distintos períodos no se elabora considerando los nexos entre los tres programas básicos de la institución: docencia, investigación y extensión, ni tampoco entre las unidades y dependencias que sirven de apoyo a la ejecución del mismo. Esto conlleva en palabras de Moreno (2002:43) a que no exista una real vinculación entre el plan de la universidad y lo que ejecutan las distintas instancias, cada quien trata de hacer lo que puede dentro de sus limitaciones. Pero cabría agregar que también dentro de sus intereses.

Tal es el caso de la reforma estructural aprobada por el Consejo Universitario mediante la Resolución 454 en el período de gobierno 2000-2004 mediante la cual se aprueba el modelo matricial para todas las facultades de LUZ, sin embargo, sólo las tres facultades que fueron designadas como las encargadas de adelantar el proceso con carácter experimental hicieron algunos adelantos. Lo significante de la situación es la discrepancia entre lo planificado y lo ejecutado tomando en cuenta que fue aprobado en Consejo Universitario para toda la institución. Según Lombardi (1996:29) “...una cosa es la decisión y otra es que se cumpla esa 
Injerencia del modelo burocrático-populista en la reforma universitaria..

Pereira Burgos, Morela; Díaz Barrios, Jazmín y Díaz Nava, María Gabriela

decisión, es decir, toda nuestra estructura burocrática pareciera diseñada para que las cosas no se hagan....".La planificación de la reforma queda entonces en el papel, dando soporte y legitimidad a la gestión sin que ello afecte el status quo de la mayoría.

En lo que respecta a los mecanismos de asociación, la reforma se torna dependiente del apoyo que recibe una determinada propuesta en el Consejo Universitario, único cuerpo directivo de la estructura universitaria en su nivel superior o estratégico. La presión de los grupos políticos que detentan el poder inclina la balanza hacia una u otra alternativa o en todo caso a su aprobación o no. Es conocido por todos, los arreglos entre grupos políticos para alcanzar una mayoría que determina la decisión.

De acuerdo con Gómez y Magro (2007), las agendas de las sesiones ordinarias de los Consejos Universitarios son aprobadas por los equipos rectorales. En algunos casos se realizan reuniones formales de grupos internos (Autoridades Rectorales, Decanos, Representantes Profesorales, Representantes Estudiantiles) para acordar posiciones comunes en cuanto a puntos de interés sectorial. También hay situaciones en las cuales participan actores externos al cuerpo, representativos de grupos gremiales o políticos que coexisten en las universidades.

En cuanto a los mecanismos de regulación, se puede argumentar que los mismos en ocasiones se constituyen en un obstáculo para la consecución de la reforma, debido principalmente a la profusa, densa y desfasada normativa existente. Al respecto señalan Gómez y Magro (2007) que los aspectos legales y normativos que rigen las organizaciones universitarias en Venezuela, ostentan un desfase significativo entre los instrumentos fundamentales que rigen estas instituciones y la realidad práctica de su desarrollo. La Ley de Universidades data de 1970, por lo que los reglamentos y normativas derivados de ella no reflejan los continuos avances que se han producido en las ciencias gerenciales $y$, en particular, en lo relacionado con el diseño organizacional.

No obstante, en los cuatro períodos revisados se constata la permanente reforma en materia normativa, mediante la creación y modificación de reglamentos y otros instrumentos, lo que necesariamente no implica mejoras en los procesos internos.

Ahora bien, uno de los factores característicos del modelo burocrático-populista que impera en la institución universitaria es el Crecimiento desproporcionado experimentado a partir de la democratización del país; aumentó la matrícula estudiantil y con ella la necesidad de incorporar mayor cantidad de docentes, personal administrativo y obrero. Sin embargo, con el transcurso del tiempo se ha constituido en un elemento perverso del modelo, al erigirse como la vía para lograr una mayor asignación presupuestaria.

Según Guerra y Ponte (2003:171) la realidad burocrática de las universidades públicas venezolanas es que el $57 \%$ del personal es de apoyo administrativo y obrero, lo que significa que este tipo de función sobrepasó al personal dedicado a las labores de docencia, investigación y extensión. En la mayoría de los casos, el incremento de este personal no obedece a las necesidades organizacionales, pero las presiones de los grupos sindicales y políticos logran penetrar las esferas de 
decisión, mediante el uso de prácticas clientelares y apoyos políticos.

Este crecimiento, bajo el amparo del modelo burocrático-populista ha configurado una estructura organizativa vertical con excesivo predominio de funciones administrativas, en la generalidad de los casos desarticuladas y aisladas así como una acentuada rigidez estructural y por ende dificultad para ofrecer respuestas oportunas. Al respecto, señala Ramos (2007:19) que las consecuencias de esta configuración son, la generación de procesos obsoletos, lentos e inoportunos; desperdicio y duplicidad de esfuerzos y limitaciones para actuar con efectividad y eficacia ante las exigencias del entorno e intorno universitario.

Entre estas exigencias destacan: desarrollo científico y tecnológico, nuevas formas de producción de conocimientos, formación de profesionales y procesos de distribución social de los bienes culturales y materiales. Aunado a ello, se tiene una infraestructura física desgastada que apenas alcanza a albergar la gran cantidad de personal que confluye en ella.

En este contexto las reformas que apunten a controlar estos procesos tienden, por una parte, a ser neutralizadas, pues atentan contra los intereses de los grupos que ostentan el poder, mientras que otras son aprobadas (filtradas) para mantener la necesaria legitimidad. Al respecto, cabe señalar la reorganización gerencial-administrativa aprobada durante el período 1992-1996 y la reestructuración administrativa aprobada con la resolución 370 en el período 1996-2000.

Estas últimas puestas en práctica sin criterios claros de gestión, pues según Díaz et al. (2004), a pesar de que un alto porcentaje $(82 \%)$ de los cambios estructurales planteados fueron ejecutados, subsiste un $18 \%$ que no lo fue, lo cual significa un grave problema de gobernabilidad. Por otra parte, no todos estuvieron soportados por estudios y análisis técnicos $(30 \%)$. Igualmente se señala que la persistencia de decisiones ejecutivas y la asistematicidad en el proceso de toma de decisiones, conspiran contra un proceso de modernización en LUZ.

Otro ejemplo lo constituye la reforma normativa referida al Reglamento de Evaluación y Rendimiento Estudiantil, que sin duda permitiría hacer frente al grave problema de la permanencia de estudiantes con materias reprobadas de manera sistemática, los cuales limitan la capacidad del sistema para nuevos ingresos. Ocurre, sin embargo, que cuando en los departamentos se exige la aplicación del mismo, desde distintas instancias se esgrimen argumentos en contra de la aplicación de la medida, en tanto atenta contra los intereses de los grupos de poder que ven afectada su imagen para el futuro.

Respecto a los Criterios de Dirección, resulta pertinente el planteamiento de Casas (1996:86), quien afirma que es frecuente encontrar en las universidades una dirección universitaria que algunos autores denominan "Gerencia Ritualista", es decir, esencialmente "política", en la cual la planificación, administración y operación, obedecen más a la negociación de los dirigentes con las fuerzas políticas, que a una adecuada racionalidad; en este tipo de dirección, la racionalidad es sólo un "ritual", es sólo la forma externa que muestra la dirigencia para "mantener las apariencias".

En otras palabras los criterios se expresan en el papel, pero en la práctica que- 
Injerencia del modelo burocrático-populista en la reforma universitaria..

Pereira Burgos, Morela; Díaz Barrios, Jazmín y Díaz Nava, María Gabriela

da demostrado que son letra muerta, ejemplo de ello lo constituyen la Filosofía de Gestión del año 1994 y 1997 así como el Plan de Desarrollo Estratégico también del año 1997. Estos instrumentos constituyen documentos declarativos del "deber ser" y sirven de soporte a la redacción de la planificación estratégica en las distintas instancias universitarias, pero en la práctica son desconocidos e ignorados por los distintos actores del quehacer universitario.

Otro ejemplo de la carencia de criterios de dirección que viabilicen los procesos de ejecución en la universidad es la firma de numerosos convenios con el sector externo, sin considerar muchas veces, su pertinencia así como mecanismos de seguimiento y evaluación. De acuerdo con Primera (2005), se ha dado el caso de convenios que caducan sin ser utilizados por alguna de las partes. Adicionalmente, Montilla et al. (2005) sostienen que los convenios entre el sector académico y el sector externo se han desarrollado fundamentalmente de manera informal y no institucional.

En relación a los elementos del discurso y manejo de conflictos, resulta sobrecogedora la cantidad de discursos, textos, foros, charlas, simposios, entre otros, en los cuales de manera reiterada y sistemática se plantea la necesidad de reformar la universidad. Las propuestas identificadas en el período de estudio muestran avances en el camino de mejorar la institución mediante el cambio, sin embargo, pareciera que la retórica supera la puesta en práctica de tales propuestas. Si conviene a los intereses de la dirigencia, se enarbola como la bandera que identifica la gerencia universitaria de tur- no, caso contrario, se deja en un segundo plano a la espera de la voluntad o interés de otros gestores.

Señala Téllez (2003:240) que para unos, aunque no lo digan públicamente, la reforma universitaria consistiría en que todo cambie sin cambiar, por ejemplo, que todo cambie sin que sean afectadas las cuotas de poder que se tienen, los saberes que se imparten, las cátedras que se ocupan, los departamentos de permanencia perenne, las facultades atomizadas, las carreras establecidas, las complicidades perversas, los comportamientos autoritarios.

Al respecto, agrega Muro (2004:57) que el sistema de representaciones presente en nuestras autoridades, genera discursos anecdóticos, retóricos y superficiales, lo cual evidencia un marcado déficit teórico conceptual para conducir los procesos de transformación y cambio.

El caso de las reformas curriculares, es una evidencia palpable de un discurso que plantea cambios significativos en procesos medulares a partir de su concreción; no obstante, la historia ha demostrado que los diseños curriculares en LUZ, producto de las reformas que se hicieron en los cuatro períodos de gobierno estudiados, no alcanzaron a aplicarse en toda su amplitud y profundidad. De acuerdo con Lanz (2004:201) se sabe de antemano que los grandes esfuerzos por reformar planes de estudio -sin conexión con ajustes estructurales en el conjunto del sistema- se reducen en el mejor de los casos a mejoras funcionales en la gestión académica.

Sin duda alguna, el modelo burocrático-populista, ha determinado la gestión académico-administrativa en la Universidad del Zulia, pero también el mode- 
lo tecnocrático dejó su huella, que evidentemente fue consecuencia de los lineamientos que desde el Estado permearon al conjunto de instituciones públicas, con mayor énfasis en las décadas de los ochenta y noventa.

Como resultado, en la práctica la educación que se ha venido impartiendo pareciera subordinar la formación sensible a la problemática social, crítica y amplia, por una visión técnica e instrumental de la realidad. Tendencia que va en consonancia con el modelo económico de mercado. Según Filmus (2000:27), las consecuencias de la aplicación de ese modelo repercutieron fuertemente en la educación, ya que muchas de ellas se han convertido en verdaderos límites a la potencialidad democratizadora que presentan las transformaciones educativas.

Con esta nueva perspectiva la gestión 1992-1996 dio impulso, mediante el programa "Negociación de Ciencia y Tecnología", al Proyecto del Parque Tecnológico Universitario (PTU) con el cual se esperaba promover la función de extensión en la universidad y una mayor vinculación del profesorado con la realidad a través de sus investigaciones. Igualmente se esperaba el logro de ingresos propios mediante la producción y comercialización de bienes y servicios producidos en la universidad. Su puesta en marcha se logró en la gestión 1996-2000.

De acuerdo con Suárez y Pereira (2004), dentro de las críticas que se hacen a esta figura están en primer lugar, la escasa participación de los sectores público (Gobernación del Zulia, CORPOZULIA, FUNDACITE-Zulia) y privado (FEDECAMARAS) en los proyectos que ejecuta el PTU; en segundo lugar, la concep- ción de parque tecnológico asumida por la institución por tener como actividad principal la comercialización de productos y no la generación de nuevos conocimientos; y en tercer lugar, que las ideas emprendidas han carecido de sostenibilidad en el tiempo.

En suma, todos estos elementos del modelo de gestión burocrático-populista presentes en la universidad, han incidido en las reformas planteadas durante el periodo estudiado. La forma en la cual se han ejecutado o no estas reformas, tiene que ver con el modelo gerencial centralizado de planificación y toma de decisiones, bajo una dinámica clientelar que impregna todos los procesos y supedita el interés institucional a otro tipo de intereses.

\section{Conclusiones}

Durante las cuatro gestiones estudiadas hubo intentos por reformar la Universidad del Zulia, lo cual se muestra en los planes y proyectos que los distintos gobiernos universitarios diseñaron y ejecutaron. Sin embargo, en la práctica algunos se han diluido, pues no siempre los esfuerzos realizados durante un período rectoral tienen continuidad en el período siguiente. Las propuestas e iniciativas no terminan de concretarse y por ende tampoco se observan resultados tangibles que den muestras de un verdadero compromiso con la transformación del sector universitario.

En este panorama el modelo de gestión imperante -burocrático-populistaha sido determinante. La toma de decisiones centralizada en la cúpula organizacional (CU), con escasa participación de los distintos actores universitarios; planificación desarticulada del resto de los 
Injerencia del modelo burocrático-populista en la reforma universitaria..

Pereira Burgos, Morela; Díaz Barrios, Jazmín y Díaz Nava, María Gabriela

procesos, atomizada, carente de coordinación, lo que parece indicar que sus fines están orientados a la legitimación social y no como guía para la acción. Adicionalmente, se observa un exceso de formalización, lentitud en las respuestas, todo lo cual ha devenido en una mediatización de las reformas.

Sin duda alguna el modelo de gestión es fundamental, pero los gestores juegan un papel preponderante, ya que son los responsables de darle vida al modelo. Esta probado que la voluntad y compromiso personal de la gerencia son elementos definitivos para alcanzar el éxito de las organizaciones (y también su fracaso). En el caso de los gestores universitarios -salvo contadas excepciones- lejos de propiciar la reorientación o modificación del modelo se han aprovechado de sus disfuncionalidades, en detrimento de los intereses verdaderamente institucionales y de las mayorías.

Como resultado tenemos una institución rezagada, a pesar de todos los intentos de transformación, respecto a las transformaciones de la sociedad en la cual está inmersa, que exige de ella respuestas pertinentes y acordes con los tiempos que transcurren. El reto es impostergable la universidad autónoma necesita reformarse, la toma de conciencia de sus actores es urgente, el rescate de la conciencia crítica y reflexiva alejada de la postura política es indispensable, es preciso que cada actor recobre y/o desarrolle, su identificación con la institución.

Pero por sobre todo: el problema es de gerencia. Es necesario un cambio en la actitud de todos y cada uno de los ge- rentes universitarios, deben, por una parte, honesta y abiertamente desear el cambio de la Universidad, y por la otra, liderar el proceso que sume voluntades hacia abajo y convierta las propuestas de reformas en realidades tangibles. De esta manera, se dará la necesaria alineación de la Universidad con su entorno, tal como la sociedad lo está exigiendo.

\section{Referencias bibliográficas}

Burbano de Lara, Felipe (1998). El fantasma del populismo. Aproximación a un tema (siempre) actual. Instituto Latinoamericano de Investigaciones Sociales-ILDIS. Facultad latinoamericana de Ciencias Sociales-FLACSO. Editorial Nueva Sociedad. Caracas, Venezuela. Pág. 228.

Casas Armengol, Miguel (1996). Especificidad de la universidad venezolana autónoma. En Universidad Utopía y Praxis. Compilador Ángel Lombardi. Universidad del Zulia. Maracaibo, Venezuela. Pág. 80-90.

Chiavenato, Idalberto (2001). Administración. Proceso Administrativo. Ediciones MacGraw-Hill Interamericana, S.A. Tercera Edición. Bogotá, Colombia.

Consejo Universitario (1988-2004). Gacetas Universitarias. La Universidad del Zulia. Maracaibo, Venezuela.

Consejo Universitario (1999). Resolución 370. Universidad del Zulia. Maracaibo, Venezuela.

Consejo Universitario (2003). Resolución 454. Universidad del Zulia. Maracaibo, Venezuela.

Congreso de la República de Venezuela (1970). Ley de Universidades. Gaceta Oficial Extraordinaria № 1429.

Díaz Barrios, Jazmín; Annicchiarico, Elvira y Borjas, Carlos (2004). Evaluación de 
la Estructura Organizativa de la Administración Central de la Universidad del Zulia. Revista TELOS, Vol. 6, No. 3. Universidad Rafael Belloso Chacín, Maracaibo, Venezuela, Pp. 392-406.

Diccionario de la Real Academia Española (2001). 22. ${ }^{a}$ Edición Disponible en: http://buscon.rae.es/drael/SrvltConsulta?TIPO_BUS=3\&LEMA=cultura. Consultado el 13.12.09.

Esté, Nina (1998). La educación superior venezolana. Una institución en crisis. Testimonios orales de sus actores. Universidad Central de Venezuela. Consejo de Desarrollo Científico y Humanístico. Caracas, Venezuela. Pág. 156.

Fergusson, Alex (2003). Relevamiento de experiencias de reformas universitarias en Venezuela. Proyecto IESALC/UNESCO. Disponible en: www2.iesalc.unesco.org.ve:2222/

.../reformas/.../Informe\%20Reformas \%20Venezuela\%20-\%20Final.pdf.

Filmus, Daniel (2000). Educación y desigualdad en América Latina de los '90 ¿Una nueva década perdida? En: La educación en el horizonte del siglo XXI. Coordinadores: Carlos Tünnermann B. y Francisco López Segrera. Colección respuestas. Ediciones IESALC-UNESCO. Caracas, Venezuela.

French, Wendel L. y Bell Jr., Cecil H. (1996). Desarrollo Organizacional. Aportaciones de la conducta para el mejoramiento de la organización. Prentice-Hall Hispanoamericana, S.A. Quinta edición. México. Pág. 442.

Gómez Zamudio, José y Magro Ramírez, Marcela (2007). Procesos decisorios de postgrado en universidades de Venezuela. El caso de dos universidades públicas. Paradigma, Vol. 28, No.1. Pág. 39-64.

Guerra, Alexis y Ponte de Moreno, Beatriz (2003). UCLA: expresiones de un proceso de reforma. En: La Universidad se Reforma. Compilador Rigoberto Lanz. Universidad Central de Venezuela. Observatorio Internacional de Reformas Universitarias (ORUS). Instituto Internacional de la UNESCO para la Educación Superior en América Latina y el Caribe (IESALAC). UPEL. Ministerio de Educación Superior. Caracas, Venezuela. Pp. 163-199.

Guerrero, Omar (2006). Tecnocracia o el fin de la política. Universidad Nacional Autónoma de México. Instituto de Investigaciones Jurídicas. Serie Doctrina Jurídica, Num. 287. México.

Hussey, David E. (1997). Como administrar el cambio en la organización. Editorial Panorama. Pág. 107.

Kelly, Janet (2004). Políticas públicas en América Latina. Teoría y Práctica. Ediciones IESA. Caracas, Venezuela.

Lanz, Rigoberto (2004). Reformar es lo más difícil. En: La Universidad se Reforma II. Compilador Rigoberto Lanz. Universidad Central de Venezuela. Observatorio Internacional de Reformas Universitarias (ORUS). Instituto Internacional de la UNESCO para la Educación Superior en América Latina y el Caribe (IESALAC). UPEL. Ministerio de Educación Superior. Caracas, Venezuela. Pp. 199-215.

Lanz, Rigoberto y Fergusson, Alex (2005). La Reforma Universitaria en el contexto de la mundialización del conocimiento (Documento rector). Observatorio Internacional de Reformas Universitarias (ORUS.Int.) Disponible en: http://www.debatecultural.net/Observatorio/RigobertoLanz22.htm

Limdblom, Charles E. (1991). El Proceso de Elaboración de Políticas Públicas. Colección: Estudios. Serie: Administración General. Ministerio para las Administraciones Públicas. Traducción: Eduardo Zapico Goñi. $1^{\text {era }}$ edición. Madrid. 
Injerencia del modelo burocrático-populista en la reforma universitaria...

Pereira Burgos, Morela; Díaz Barrios, Jazmín y Díaz Nava, María Gabriela

Lombardi, Ángel (1996). Conferencia central del Dr. Ángel Lombardi. En: Universidad. Teoría y Praxis. Encuentro Nacional de la Universidad, su Concepción y Praxis. Colección Reapertura. Universidad del Zulia. Pp. 17-34.

López Sánchez, Roberto (1996). Universidad, Política y Cultura. Años 60 vs. Años 90. Revista Opción, No. 20. Universidad del Zulia. Maracaibo, Venezuela. Pp. 53-71.

Malave, José y Piñango, Ramon (2004). La Organización del Estado y las Políticas Públicas. En: Políticas Públicas en América Latina. Teoría y Práctica. Coordinadora Janet Kelly. Ediciones IESA. Caracas, Venezuela. Pp. 2958.

Montilla, Aída; Prieto, Letícia; Arenas, Olga, Colina, Belinda y Abreu Nerio (2005). Modalidad convenio de cooperación de la Universidad del Zulia. Contexto teórico-metodológico. Simposio Ciencia y Desarrollo Endógeno ¿Viejos Términos y/o Nuevas Concepciones? Caracas. Disponible en: http://www.saber.ula. Ve/bitacora-e/eventos/resumenes_ simposio_20al25-11-05.html

Mora, Julia (1999). Transformación y gestión curricular. En: Memorias Seminario Taller Evaluación y Gestión Curricular, Universidad de Antioquia.

Moreno Peinado, Emilio (2002). Gaceta Universitaria. Volumen I. Enero-Marzo. Maracaibo, Venezuela. Pág. 43.

Morles, Víctor; Medina Rubio, Eduardo y Álvarez Bedoya, Neptalí (2003). La Educación Superior en Venezuela. Disponible en: http//wwwiesalc. UNESCO.org.ve/pruebaobservatorio/Docentes\%20formatos\%20varios/ ES-VZLA-TEXTO-NOV2002-2.PDF.I 25/04/03.

Muro, Xiomara (2004). La transformación universitaria desde el discurso ofi- cial y el discurso de las autoridades universitarias. En: La Universidad se Reforma II. Compilador Rigoberto Lanz. Universidad Central de Venezuela. Observatorio Internacional de Reformas Universitarias (ORUS). Instituto Internacional de la UNESCO para la Educación Superior en América Latina y el Caribe (IESALAC). UPEL. Ministerio de Educación Superior. Caracas, Venezuela. Pp. 47-61.

Ochoa, Haydeé (1995). Tecnocracia y Empresas Públicas en Venezuela (1948-1991). Universidad del Zulia. Vicerrectorado Académico. EDILUZ. Maracaibo, Venezuela.

Ochoa Henríquez, Haydee; López Valladares, Mirtha y Rodríguez Colmenares, Isabel (1996). Administración pública y populismo en Venezuela. Revista Venezolana de Gerencia, Vol. 1, No.1. Universidad del Zulia. Maracaibo. Pp. 39-58.

Ochoa Ávila, Migdely; Valés Soa, Mario y Quevedo Aballe, Yonanni (2007). Innovación, tecnología y gestión tecnológica. Revista Cubana de los profesionales de la información y de la comunicación en salud (ACIMED). Volumen 16, número 4.

Parra, María Cristina (1996). Los profesores universitarios en un proceso de transición, Un estudio de caso: Los profesores de la Universidad del Zulia. Revista Espacio Abierto, Cuaderno Venezolano de Sociología, Vol. 5, N ${ }^{\circ}$. 3. Septiembre-Diciembre, Pp. 470-493.

Pereira de Homes, Lilia, Díaz Barrios, Jazmín, Pereira Burgos, Morela Pereira y Suárez, Wendolin (2008).Influencia del Modelo Burocrático-Populista en la Gestión de la Investigación: Caso Universidad del Zulia. Revista de Ciencias Sociales de la Universidad del Zulia. Vol. XIV Nº 3. Sept-Dic. 
Primera, Marlene (2005). Entrevista realizada el 12.05.05 en su condición de Directora de Relaciones Interinstitucionales de la Universidad del Zulia. Maracaibo, Venezuela.

Ramos, César (2007). Universidades, cultura, innovación y vinculación con el entorno socioproductivo. Colección Textos Universitarios. Ediciones del Vicerrectorado Académico. Universidad del Zulia. Maracaibo, Venezuela. Pág. 136.

Suárez, Wendolin y Pereira, Lilia (2004). Política de vinculación con el sector productivo a través del Parque Tecnológico de la Universidad del Zulia. Ponencia presentada en el 54 Convención Anual de la Asociación Venezolana para el Avance de la Ciencia celebrado en Valencia del 14 al 21 de noviembre.

Subirats, Joan (1989). Análisis de Políticas Públicas y Eficacia de la Administración. Instituto de Administración Pública. Madrid, España.
Téllez, Magaldy (2003). La reforma universitaria: ¿con vistas a qué? En: La Universidad se Reforma. Compilador Rigoberto Lanz. Universidad Central de Venezuela. Observatorio Internacional de Reformas Universitarias (ORUS). Instituto Internacional de la UNESCO para la Educación Superior en América Latina y el Caribe (IESALAC). UPEL. Ministerio de Educación Superior. Caracas, Venezuela. Pp. 239-273.

Universidad del Zulia (1994). Filosofía de Gestión de la Universidad del Zulia. Ediciones Astro Data, Maracaibo, Venezuela.

Universidad del Zulia (1997). Filosofía de Gestión de la Universidad del Zulia. Ediciones Astro Data, Maracaibo, Venezuela.

Universidad del Zulia (1997). Plan de Desarrollo Estratégico. Maracaibo, Venezuela.

Weber, Max (1964). Economía y Sociedad. México: Fondo de Cultura Económica. 